\title{
LncRNA DLEU2 regulates Sirtuins and mitochondrial respiratory chain complex IV: a novel pathway in obesity and DOHaD
}

Jian Zhang, Matthew Krivacka Kay, Mahua Choudhury*

Pharmaceutical Sciences, Texas A \& M Health Science Center

*Correspondence: mchoudhury@tamu.edu

\section{HIGHLIGHTS}

Maternal diet can modulate hepatic IncRNA DLEU2 and its downstream regulators in offspring

DLEU2 is required for specific sirtuin regulation and mitochondrial respiration chain complex IV expression

Offspring liver depleted of DLEU2 show decreased mitochondrial respiration chain complex IV and specific sirtuins at transcriptional and translational levels

\section{ABSTRACT}

Long noncoding RNAs (IncRNAs) are commonly dysregulated in cancer but are mostly unknown for roles in metabolic regulation. Sirtuins, an epigenetic modulator class, regulate metabolic pathways. However, how sirtuins are regulated via IncRNA is unknown. In our findings, DLEU2, a IncRNA involved in primarily blood cancers, and sirtuins were both significantly decreased in the livers of high-fat high-fructose diet (HFD-HF) fed male mouse offspring (F1) whose mothers (F0) were either fed chow diet or HFD-HF during reproductive and pregnancy windows. Confirming this connection, upon silencing DLEU2, transcription levels of SIRT1 through 6 and translational levels of SIRT1, 3, 5, and 6 were significantly downregulated. Knockdown of DLEU2 also significantly decreased the protein level of cytochrome-c oxidase (complex IV, MTCO1) 
without altering other mitochondrial complexes, leading to increased reactive oxygen species production. Interestingly, in F1 livers, the protein level of MTCO1 was also significantly decreased under an HFD-HF diet or even under chow diet if the mother was exposed to HFD-HF. Our findings therefore reveal for the first time that a IncRNA can regulate sirtuins and a specific mitochondrial complex, furthermore suggesting a potential role of DLEU2 in metabolic disorders over one or more generations.

\section{INTRODUCTION}

Obesity has emerged as the leading medical disorder of the 21 st century with an ever increasing incidence throughout the world ( $\mathrm{Ng}$ et al., 2014). Several factors have been pinpointed for this increase in obesity; for example, a sedentary lifestyle and improper dietary habits, including a Western diet (Soro-Arnaiz et al., 2016). Western diets, in particular those high in carbohydrates and fats, have led to a procession of obesityrelated disorders, including type 2 diabetes, dysglycemia, and metabolic syndrome (Taylor and Poston, 2007). This obesity epidemic is even more severe than at first appearance, as there is increasing evidence showing that exposure to a Western diet to the mother increases the risks of offspring developing features of metabolic syndrome as well, known as the "Developmental Origins of Health and Disease" (DOHaD) theory (Godfrey, 1998; Jones et al., 2009; King, 2006; Samuelsson et al., 2008; Yajnik and Deshmukh, 2008). As the impacts of obesity can pass from one generation to the next, scientists are now investigating how and why these signals for metabolic dysregulation can be inherited. Epigenetic inheritance can play a broad role in enacting these scenarios (Choudhury and Friedman, 2011; King and Skinner, 2020; Li, 2018). 
Epigenetics involves the heritable transmission of phenotype changes which do not involve alterations in the DNA sequence (Dupont et al., 2009). These transmissible changes can be through long non-coding RNAs (IncRNAs), which are non-coding RNA fragments known to be more than 200 nucleotides long (An et al., 2017; Ma et al., 2013; Perkel, 2013). IncRNAs were originally considered irrelevant transcriptional noise, but have since been shown to play key roles in both physiological and pathological regulatory pathways (Mazo et al., 2007; Rinn et al., 2007). DLEU2, short for Deleted in leukemia 2, is a recently discovered IncRNA which has been shown to play an important role in multiple cancers, including blood (Klein et al., 2010), renal (Lv et al., 2017), and pancreatic (Xu et al., 2019). DLEU2 IncRNA also serves as the intronic site of tumor suppressor microRNAs (miRNAs) miR-15a and miR-16-1, which play downstream roles in controlling cell cycling functions (Lerner et al., 2009). Of considerable note, miR-15a and miR-16-1 have been shown to be involved in metabolic regulation, including insulin signaling and PI3K-Akt pathways (Kwon et al., 2014). However, no connections have been drawn between the IncRNA DLEU2, metabolic disorders, and obesity as of yet.

Epigenetic regulation can also be applied through histone and protein molecular tagging, such as lysine acetylation/deacetylation of terminal residues, to control gene accessibility and expression (Choudhary et al., 2009; Hirschey et al., 2011; Kendrick et al., 2011; Milazzo et al., 2020). Sirtuins, a seven-membered family (SIRT1-7) of epigenetic regulators, located in different subcellular regions, are grouped as the class III histone deacetylases (HDACs) (Feldman et al., 2012). The sirtuins (SIRTs) are separated by their activities, with SIRT1, 2, 6, and 7 located in the nucleus and SIRT 3, 4, and 5 located in the mitochondria (Feldman et al., 2012). Our group and others show 
that SIRTs display crucial roles in a variety of key metabolic pathways, including energy metabolism, stress response, fatty acid oxidation, triglyceride pathways, inflammation, and mitochondrial regulation (Borengasser et al., 2011; Choudhury et al., 2011;

Kendrick et al., 2011; Suter et al., 2012; Zhang et al., 2015). SIRTs thus participate in a variety of aspects, controlling metabolism and stress throughout the body. There are no reports yet connecting all these sirtuin regulations with any specific IncRNA. Interestingly, high-Myc state repressed DLEU2 and Myc binding site was shown to be associated with DLEU2's promoter region (Chang et al., 2008). While no direct pathways have been implicated, interplay has been shown between sirtuins and Myc cancer-related metabolic reprogramming (Zwaans and Lombard, 2014). Recent evidence has also shown that some HDACs inhibitors, such as trichostatin A (TSA), upregulate DLEU2 and miR-15a/16-1, in lung cancer cells (Chen et al., 2013). However, though cancer links have been discovered, to our knowledge no studies have been performed linking any IncRNAs with sirtuins, especially via dietary factors. Herein we hypothesize DLEU2 may regulate sirtuins.

Sirtuins have been shown to play a major role in mitochondrial modulation and regulation (Carrico et al., 2018; Hirschey et al., 2011; Nasrin et al., 2010). Mitochondria play key roles in maintaining homeostasis by generating ATP, and performing fatty acid oxidation and oxidative phosphorylation (Lombard et al., 2011). The inner mitochondrial membrane (IMM) contains the electron transport chain complexes I-IV, which generate the electrochemical gradient across the IMM. Defects and alterations in mitochondrial complex expression have been linked to metabolic syndrome and obesity (Ngo et al., 2019; Soro-Arnaiz et al., 2016; Sverdlov et al., 2015). As the mitochondria is directly 
involved in detoxifying radical oxygen species (ROS) generated from fatty acid oxidation, SIRT3 plays key roles by deacetylating mitochondrial superoxide dismutase (SOD2), inducing SOD2 activity (Qiu et al., 2010), deacetylating FOXO3 for transcription (Tseng et al., 2013), and similarly inducing the glutathione and thioredoxin systems (Kong et al., 2010; Potthast et al., 2017). As such, SIRTs have been identified as vital players in regulating both metabolic and mitochondrial factors in the body, but their links to any IncRNA regulation remain unknown.

In this study, we explore a mechanistic regulation of DLEU2 on sirtuins, the mitochondrial complex, and oxidative stress in liver cells.

\section{RESULTS}

\section{HFD-HF intake increased body weight and liver weight}

To investigate the effect of maternal HFD-HF intake on the body weight (BW) and liver weight (LW) of the offspring, measurements of BW and LW were taken at 20 weeks. Maternal high-fat high fructose intake has no effect on both the BW and LW in F1 pups (Fig. 1 A\&B, red vs blue or purple vs green). However, the body and liver weight were significantly increased in pups who were fed HFD-HF compared to CD (Fig. 1A\&B, red vs purple or blue vs green, $p<0.001$, respectively).

\section{HFD-HF intake altered hepatic gene expression of DLEU2 and SIRT}


Till now, only one article shows the intergenerational effects of HFD on IncRNAs in an array analysis, however, no in depth analysis was carried out on individual level (An et al., 2017). Our study is the first attempt to investigate the intergenerational effect of diet on DLEU2 and sirtuins. In the CD fed F1 offspring, hepatic RNA levels of DLEU2, SIRT1,

2, 4, 5, and 6 were significantly decreased when mother was fed HFD-HF compared to CD fed mother (Fig. 1, C, D, E, G, H, \&l, purple vs green $P<0.001, P<0.05, P<0.01$, $P<0.05, P<0.01$, and $P<0.01$, respectively). This showed the intergenerational effects of HFD-HF in our study. However, even in groups with mother on CD (blue and green), gene expression of DLEU2 and all sirtuins were significantly downregulated in offspring fed HFD-HF compared to pups on CD (Fig. 1, blue vs green, One-way ANOVA). These significant effects were not seen for SIRT2, 3, 4, and 5 in offspring with mother on HFDHF (Fig. $1 \mathrm{E}, \mathrm{F}, \mathrm{G}, \& \mathrm{H}$, red vs purple, One-way ANOVA or t-test). These differences indicate the different effects of maternal diet and pup's own diet.

\section{Silencing DLEU2 altered SIRT genes and proteins expression in HepG2 cells}

Our in vivo study strengthens our hypothesis that DLEU2 may regulate SIRT expression.

Therefore, we carried out an investigation of the mechanistic interaction of DLEU2 and Sirtuins in the HepG2 cell line. After DLEU2 was silenced, RNA expression of DLEU2, SIRT1 to 6 were significantly downregulated, but SIRT7 was significantly upregulated (Fig. 2). These results are in line with the above in vivo mouse study, except for SIRT7. Consistent with RNA expression, the protein levels of SIRT1, 3, 5, and 6 were decreased after DLEU2 knockdown, however, protein levels of SIRT 2, 4, and 7 were not altered (Fig. 2). These results indicated that DLEU2 may have a direct relationship with SIRT1, 3,5 , and 6 . 


\section{DLEU2 knockdown decreased Oxphos Complex IV}

Sirtuins play roles in mitochondrial function and are involved in oxphos complexes regulation (Verdin et al., 2010), indicating DLEU2 may also play a role in regulating mitochondrial function directly or indirectly. Therefore, mitochondrial complex protein levels were investigated in DLEU2 silenced cells. Interestingly, protein levels of complex IV-MTCO1 was significantly decreased after DLEU2 KD (Fig. 3, A\&E, P<0.001) while other mitochondrial complexes were unchanged.

HFD-HF intake in F0 mother altered hepatic protein expression of MTCO1 in F1 generation

Hepatic protein level of MTCO1 in F1 was investigated. Maternal HFD-HF significantly decreased pups' MTCO1 level compared to CD fed mother ( $P=0.0072$, t-test, purple vs green, Fig. $3 \mathrm{G \& H}$ ). In F1, HFD-HF feeding also decreased hepatic MTCO1 compared to $C D$ diet $\left({ }^{* *} P<0.01\right.$, blue vs green, and ${ }^{\wedge} P<0.05$, red vs purple, Fig. $\left.3 \mathrm{G} \& H\right)$.

\section{Silencing DLEU2 increased oxidative stress}

Both sirtuins and mitochondrial complex were shown to contribute in oxidative stress regulation. Oxidative stress may indicate mitochondrial dysfunction (Nita and Grzybowski, 2016). In this study, ROS levels were significantly increased in DLEU2 KD (Fig. 2Q, P < $0.01)$. 


\section{FIGURE LEGEND}

Figure 1. Maternal diet effect on weight of body and liver, and gene expression of DLEU2 and Sirtuins in the livers of F1 male mice on CD and HFD-HF.

Body (A) and liver (B) weight of F1 male pups from 4 groups were measured. CD mothers/CD pups, green; CD mothers/HFD-HF pups, blue; HFD-HF mothers/CD pups, purple; HFD-HF mothers/HFD-HF pups, red. Fold change of hepatic RNA expression of C) DLEU2, D) SIRT1, E) SIRT2, F) SIRT3, G) SIRT4, H) SIRT5, I) SIRT6, and J) SIRT7 are shown. Expression was normalized to ß-Actin. $n=6$ mice/group, one-way ANOVA with Tukey's post hoc test, ${ }^{*} \mathrm{P}<0.05,{ }^{* *} \mathrm{P}<0.01,{ }^{* * *} \mathrm{P}<0.001$ compared to $\mathrm{CD} / \mathrm{CD}$. ${ }^{\#} \mathrm{P}<0.05$ compared to CD/HFD-HF, ${ }^{\wedge} \mathrm{P}<0.01$ compared to HFD-HF/HFD-HF. The $p$ value over comparison line in the figure indicates t-test.

Figure 2. Expressions of DLEU2 and Sirtuins expression and oxidative stress in HepG2 human cancer cell lines after silencing DLEU2

In HepG2 cells, DLEU2 was knocked down for $96 \mathrm{~h}$, and gene expressions of DLEU2 and Sirtuins were analyzed by RT-PCR. RNA levels of A) DLEU2, B) SIRT1, C) SIRT2, D) SIRT3, E) SIRT4, F) SIRT5, G) SIRT6 and H) SIRT7 in DLEU2 knockdown HepG2 cells. Expression was normalized to ß-Actin. $n=3$. ${ }^{* *} P<0.01$, ${ }^{* * *} P<0.001$ versus negative control.

After DLEU2 was knocked down for $96 \mathrm{~h}$, protein expressions of sirtuins were analyzed by western blot. I) ß-Actin was used as a loading control. Densitometry analysis was performed on J) SIRT1, K) SIRT2, L) SIRT3, M) SIRT4, N) SIRT5, O) SIRT6, and P) 
SIRT7 expression. Expression was normalized to ß-Actin. $n=3,{ }^{*} P<0.05,{ }^{*} P<0.01$, ${ }^{* * *} \mathrm{P}<0.001$ versus negative control. Q) DLEU2 KD increased ROS level in HepG2 cells. Reactive oxygen species (ROS) levels were determined by measuring oxidized dichlorofluorescein (DCF) levels using a 2', 7'-dichlorofluorescein diacetate (DCFDA) after DLEU2 KD. ${ }^{* *} \mathrm{P}<0.01$ versus negative control; $\mathrm{n}=3$.

Figure 3. Expressions of OXPHOS in HepG2 human cancer cell line after silencing of DLEU2 and maternal diet effect on protein level of MTCO1 in the livers of F1 male mice on CD and HFD-HF.

A) The representative bands by western blot analysis demonstrate the protein levels of OXPHOS complexes B) CI-NDUFB8, C) CII-SDHB, D) CIII-UQCRC2, E) CIV-MTCO1, F) CV-ATP5A after $96 \mathrm{~h}$ transfection with Negative control and DLEU2 siRNA. $\mathrm{n}=6$, ${ }^{* * *} \mathrm{P}<0.001$ versus negative control. G, H) Protein level of hepatic MTCO1 in F1 liver. Expression was normalized to ß-Actin. $n=3$ mice/group, one-way ANOVA with Tukey's post hoc test, ${ }^{* *} \mathrm{P}<0.01,{ }^{* * *} \mathrm{P}<0.001$ compared to $\mathrm{CD} / \mathrm{CD} .{ }^{\wedge} \mathrm{P}<0.05$ compared to HFDHF/HFD-HF. The $p$ value over comparison line in the figure indicates t-test.

Figure 4. Proposed model of HFD-HF leading to IncRNA DLEU2 regulated epigenetic circuit causing metabolic abnormalities in the liver. Western diet shows effects on DLEU2 induced metabolic regulation leading to mitochondrial dysfunction and oxidative stress in liver. These abnormalities can lead to metabolic diseases including diabetes, obesity, fatty liver and many more. This figure was created with BioRender.com. 
Fig 1
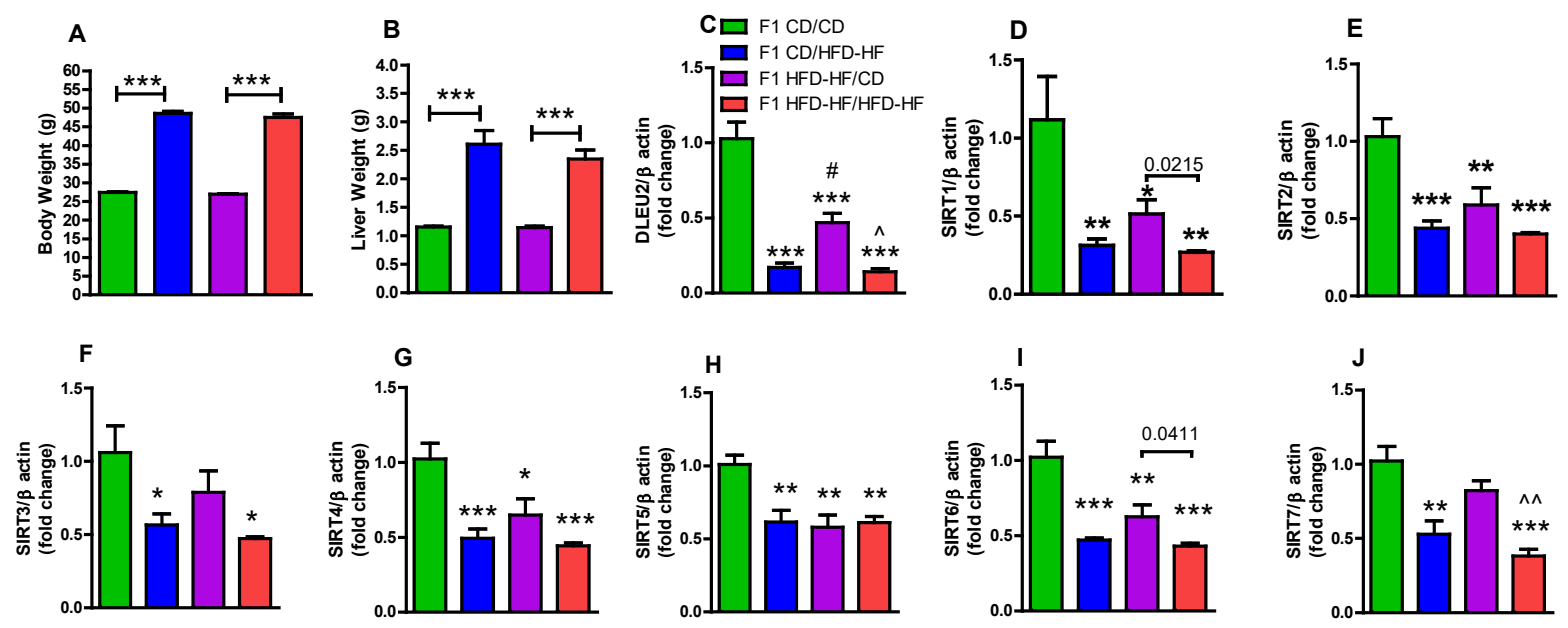

Fig 2
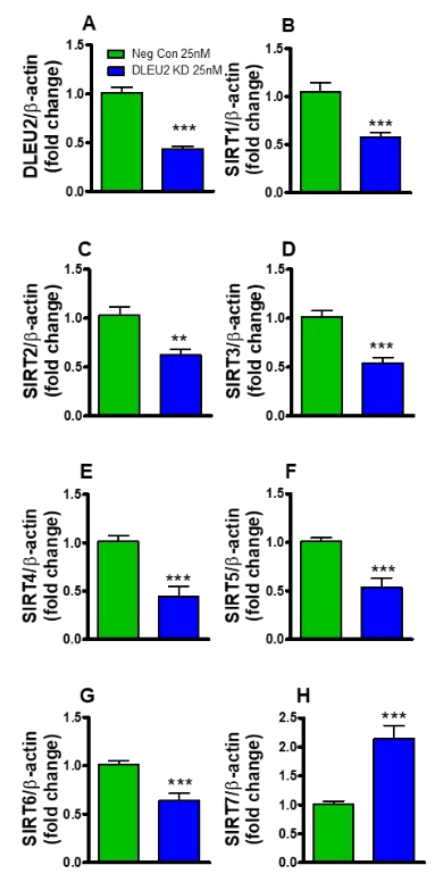

I
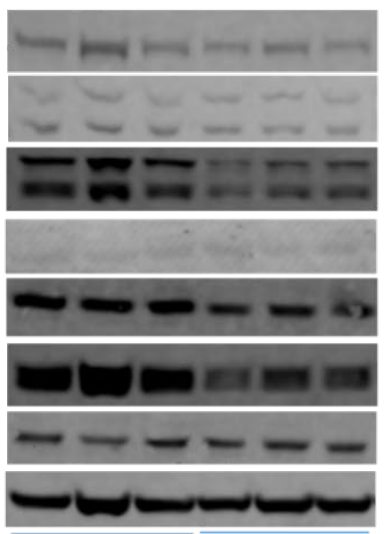

Neg 25nM DLEU2 KD 25nM
SIRT1-120KD

SIRT2-39, 43KD

SIRT3-28KD

SIRT4-32KD

SIRT5-30KD

SIRT6-42KD

SIRT7-45KD

$\beta$-actin-45KD
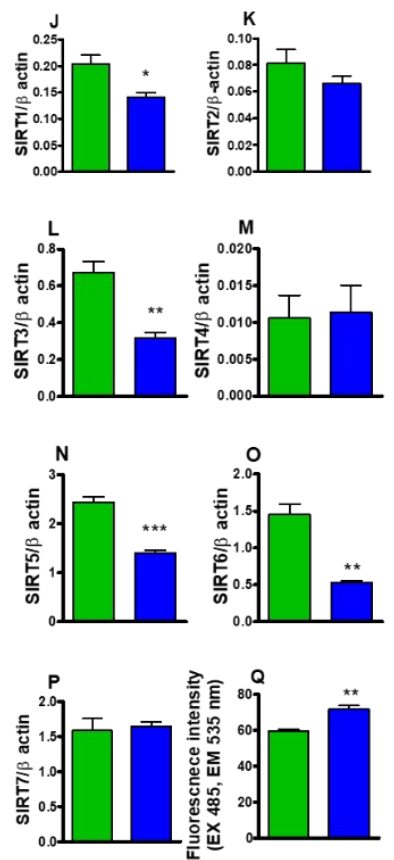
Fig 3

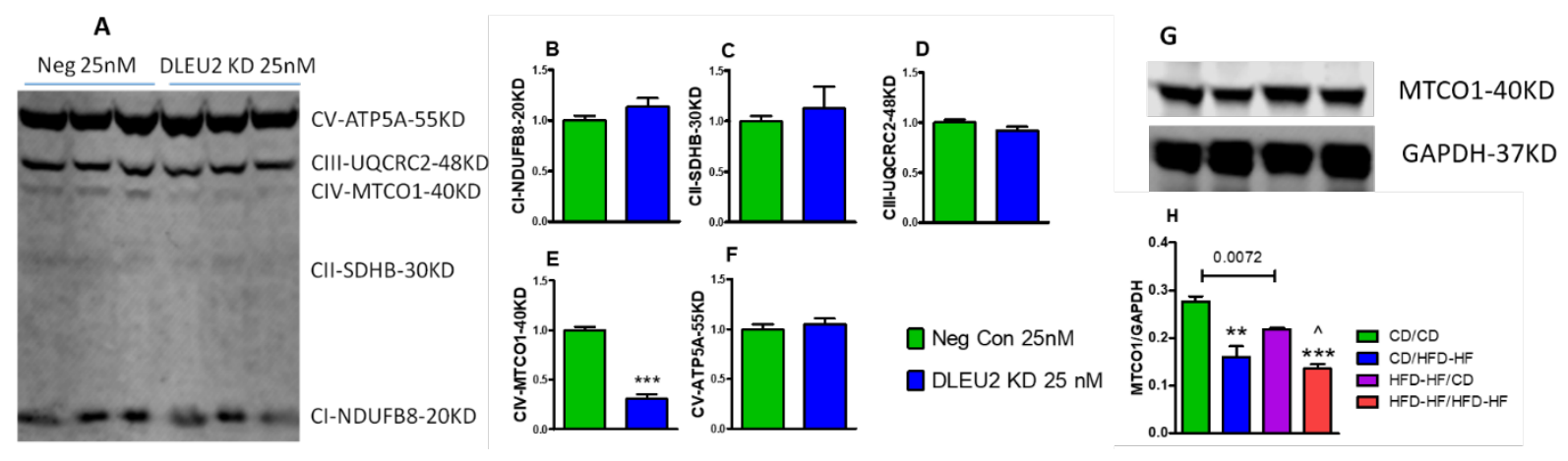

Fig 4

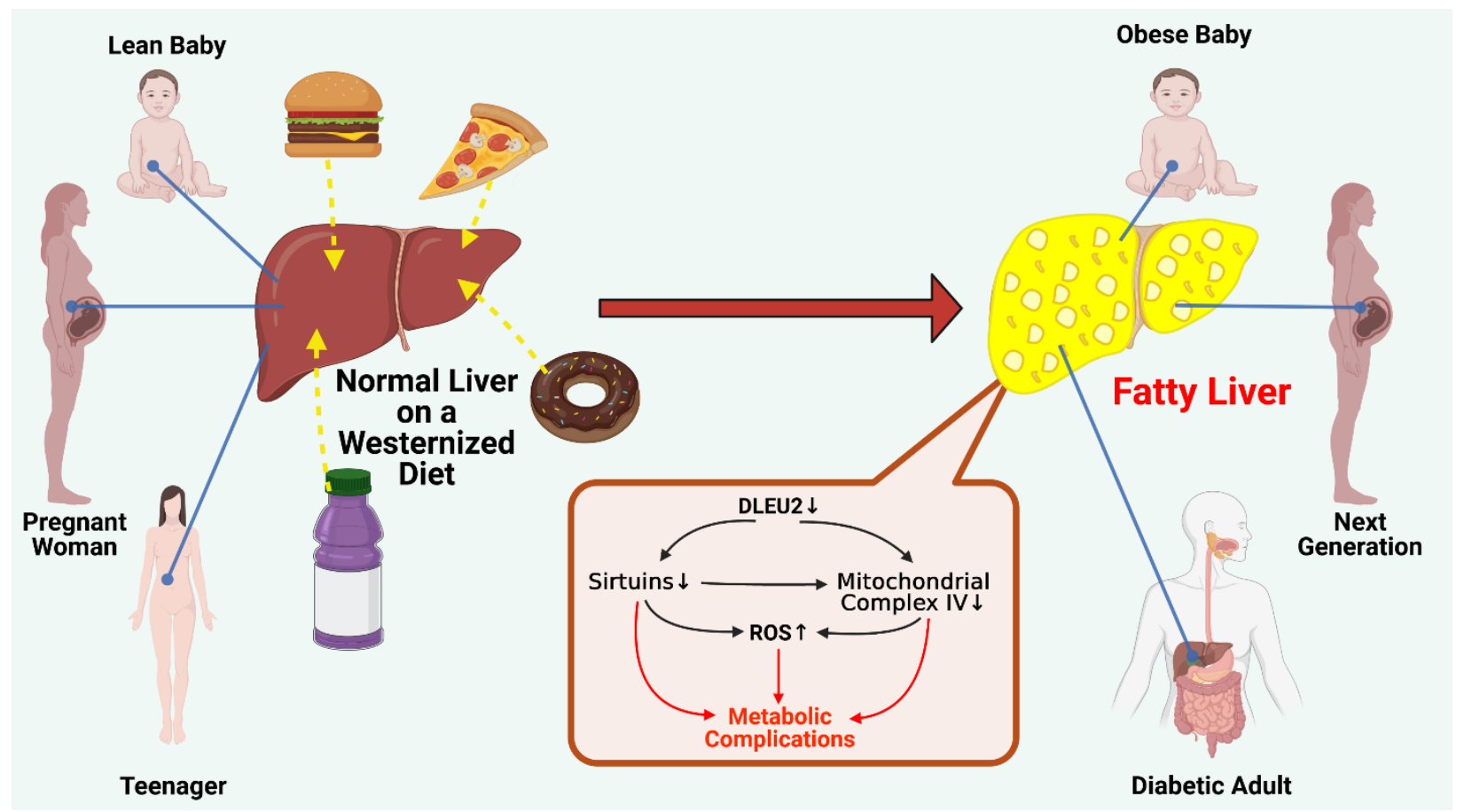




\section{DISCUSSION}

The obesity epidemic is propagated on metabolic syndrome brought about by the worldwide spread of the western diet, with its high fat content and industry-processed sugars and carbohydrates, including fructose, which causes insulin resistance, cardiovascular disease (CVD), and metabolic syndrome-related dysregulation (Abdelmalek MF, 2010; Gotto AM Jr, 2006; Lustig RH, 2016). This problem has become especially acute, as countries that have switched to high fructose corn syrup (HFCS) in their food supply have a $20 \%$ higher incidence of diabetes compared to countries that do not use HFCS (Goran MI, 2013). This diet-based premise seems to hold true, as increased weights for both the body and liver were present in our study's mice feeding on a HFD diet containing high fructose (Fig 1, blue and red bars), as would be expected from multiple related animal studies on HFD with fructose (Charlton et al., 2011; Chukijrungroat et al., 2017; Della Vedova et al., 2016; Liu et al., 2018; Love et al., 2017; Luo et al., 2016; Mastrocola et al., 2018).

It is also well known that alterations in fetal life due to changes in the in utero environment may influence the adult onset of metabolic disease as well (Godfrey, 1998; Jones et al., 2009; King, 2006; Samuelsson et al., 2008; Yajnik and Deshmukh, 2008). The $\mathrm{DOHaD}$ concept has existed for many years, and has definitively shown that changes to diet and environmental stress during gestation leads to later downstream effects in offspring later in life (Roseboom et al., 2001; Wadhwa et al., 2009). However, our group did not see an in utero maternal HFD-HF exposure effect on offspring weight (Fig 1, green and purple vs. blue and red bars). One explanation could be that in utero exposure time was not enough to fully affect the offspring's phenotypes yet at the time 
of mice sacrifice. However, evidence says that epigenetic changes happen before any symptoms arise. Therefore, it is not unlikely that there may be molecular abnormalities already initiated. As these DOHaD effects can be transmitted epigenetically (Wadhwa et al., 2009), we investigated the various epigenetic changes as these generally happen before actual genetic or phenotype changes which can functionally be reversed (Lacal and Ventura, 2018).

Epigenetic changes are suggested to be introduced from the parents to the offspring via a variety of mechanisms, including DNA methylation, posttranslational histone modification, chromatin remodeling, and non-coding RNA (Horsthemke, 2018; van Otterdijk and Michels, 2016). However, virtually no data are currently available on the role of any specific long non-coding RNA involved in metabolic dysfunction in generational inheritance.

IncRNAs are an under-investigated area in the field of metabolic epigenetics, as most of the current research is being performed in cancer related topics, with a modicum of obesity and diabetes-related subjects. Gonzalez-Rodriguez et al. discovered that heritable growth restriction was associated with changes in IncRNA H19 expression, but such changes to $\mathrm{H} 19$ expression were reversible with diet supplementation combating metabolic syndrome (Gonzalez-Rodriguez et al., 2016). As such, we investigated if the IncRNA DLEU2 would have expression changes when exposed to an obesity-causing diet regimen from mother to offspring. The change of DLEU2 in offspring under different diet was also investigated. More importantly, there are virtually no studies connecting DLEU2 with metabolic diseases. DLEU2 expression was found to be significantly decreased in the livers of offspring mice in a likewise manner, with more than $70 \%$ 
downregulated DLEU2 expression in offspring on a HFD-HF compared to on a CD (Fig $1 \mathrm{C},>80 \%$ down blue vs green and, $>70 \%$ down red vs purple bars). These specific IncRNA responses to diets led us to investigate other epigenetic modulators that might be regulated by DLEU2. A study by Chen et al. showed that the expression of DLEU2 was found to be increased by the histone deacetylase (HDAC) inhibitor trichostatin A (TSA) in non-small cell lung cancer (Chen et al., 2013). This related finding triggered our interest in searching for a specific DLEU2-HDAC relationship, specifically sirtuin interactions, as we and others have previously established this HDACIII group's role in metabolic diseases (Choudhury et al., 2011; Kendrick et al., 2011; Zhang and Choudhury, 2017; Zhang et al., 2015).

While the mice offspring exposed to western diet showed significant downregulation of sirtuins (Figure 1D-J, blue and red bars), we found that a maternal HFD-HF decreased hepatic sirtuin expression in liver of offspring. (Figure 1D, E, G, H, and I, purple bars). This result is in support of our previous findings, as our non-human primate study showed that a maternal HFD decreased the hepatic expression of Sirt1 in offspring (Suter et al., 2012). We then followed-up with a mechanistic experiment, where DLEU2 was knocked down in HepG2 cells, to reveal a direct connection between IncRNA and Sirts, as the majority of the sirtuins were downregulated at the transcriptional (Fig 2A-G) and at the translational (Fig 3I, J, L, N, and O) levels. Silencing of DLEU2 in vitro had similar genes effect as in vivo HFD-HF exposure except SIRT7 (Fig 1J and Fig 2H). This effect was diminished though at the transcriptional level for SIRT 7 as well as SIRT2 and 4 (Fig $2 \mathrm{~K}, \mathrm{M}$, and P). Of note, SIRT7 has shown the opposite role compared to SIRT1 in adipogenesis (Bober et al., 2012; Fang et al., 2017). 
Overall, we can speculate that there might be other regulators which could try to normalize a DLEU2 effect in a whole body system, however, DLEU2's effects on SIRT1, 3,5 , and 6 showed similar effects at the transcription and translational levels both in vivo and in vitro (Fig $2 \mathrm{~L}$ and N). Interestingly, two of these sirtuins ( 3 and 5 ) are localized to the mitochondria (Michishita et al., 2005), and all of these sirtuins are linked to oxidative stress (Bause and Haigis, 2013; Cheng et al., 2016; Liu et al., 2013; Salminen et al., 2013; Singh et al., 2018). SIRT3 has been known to be associated with the electron transfer chain components, including controlling acetylation of complex IV (Kendrick et al., 2011), $\beta$-oxidation of fatty acids (Hirschey et al., 2010), and extramitochondrial PGC-1 $\alpha$ co-activation for mitochondrial biogenesis (Jeninga et al., 2010), with SIRT5 involved in the urea cycle for ammonia detoxification and disposal (Nakagawa et al., 2009). Therefore, we hypothesized that DLEU2 may be the noncoding epigenetic regulator which is carrying out these downstream changes in the mitochondrial complex while modulating oxidative stress.

Fulfilling our expectations, mitochondrial defects were discovered in the form of a significant mitochondrial complex IV reduction from the DLEU2 knockdown and F1 liver, leading to increased oxidative stress (Fig. $2 Q$ \& 3). We have previously shown that in SIRT3-/ mice, exposure to a HFD reduced activity of mitochondrial complexes III and IV (Kendrick et al., 2011). These results matched other studies showing higher levels of production of mitochondrial ROS under stress-inducing conditions (Kim et al., 2010; Sundaresan et al., 2009). Potthast et al discovered the inhibition of situins 1,3 and 4 activity, protein and transcriptional levels in fibroblasts from patients with mitochondrial COX IV-deficiency (Potthast et al., 2017). Our result is specifically novel as DLEU2 is 
regulating only one mitochondrial complex-therefore a specific targeted regulation will be investigated in our future obesity study using knockout and overexpression mice.

In conclusion, a western high fat high fructose diet disturbs the hepatic regulation of an epigenetic factor DLEU2, potentially leading to metabolic disorders in the same generation or in the next generation (Fig. 4). DLEU2 directly regulates most members of sirtuins and could be a new target for modulation for remedying metabolic dysfunction, reducing oxidative stress. More in vivo studies with knockout mouse models are needed to discover the function of DLEU2 in metabolic regulation pathways and also in transgenerational inheritance, especially in the liver or other metabolic tissues.

\section{MATERIAL AND METHODS}

\section{Animal studies}

Female C57BI/6 mice (F0) were either fed an ad libitum Control diet (CD (13.2\% fat, $24.6 \%$ protein, and $62.1 \%$ carbohydrates by calories, PicoLab Rodent Diet 5053; Laboratory Supply, Fort Worth, TX) or high-fat/high-fructose diet (HFD-HF; 45\% fat, 20\% protein, $17 \%$ fructose, $17 \%$ sucrose by calories, D15041701, Research Diets) for 6 weeks till birth as previously described (Powell and Choudhury, 2019). The male pups (F1) were fed CD or HFD-HF for 20 weeks. Four groups: CD/CD (CD mothers/CD pups, green), CD/HFD-HF (CD mothers/HFD-HF pups, blue), HFD-HF/CD (HFD-HF mothers/CD pups, purple), and HFD-HF/HFD-HF (HFD-HF mothers/HFD-HF pups, red) were assigned. Body weight of F1 was measured at 20 weeks. Liver was excised, weighed, and flash frozen in liquid nitrogen. Comparison of HFD-HF/HFD-HF vs CD/HFD-HF (red vs blue) or 
HFD-HF/CD vs CD/CD (purple vs green) indicated maternal diet effect (intergenerational effect), and comparison of HFD-HF/HFD-HF vs HFD-HF/CD (red vs purple) or CD/HFDHF vs CD/CD (blue vs green) indicated offspring diet effect. All procedures were approved by Texas A\&M IACUC.

\section{Cell culture and siRNA knockdown (KD) experiments}

HepG2 cells were purchased from ATCC (Manassas, VA). Cells were cultured in EMEM media supplemented with $10 \%$ fetal bovine serum, $100 \mathrm{U} / \mathrm{mL}$ penicillin, and $100 \mathrm{mg} / \mathrm{mL}$ streptomycin (Gibco, NY). The cells were maintained in a humidified incubator at $37^{\circ} \mathrm{C}$ under an atmosphere of $5 \% \mathrm{CO}_{2}$. The negative control (Ambion; n410472) or siRNA DLEU2 (Ambion; n274197) was mixed with Lipofectamine RNAiMAX (Invitrogen) according to the manufacturer's protocol in serum-free OPTI-MEM before incubation. RNA or protein was extracted after $96 \mathrm{~h}$ transfection.

\section{mRNA real-time RT-PCR}

Liver or cell RNA was extracted according to the Qiagen miRNeasy Mini Kit (Qiagen, MD, USA) protocol. Reverse transcription (RT) was carried out using the High-Capacity cDNA Reverse Transcription Kit (Life Technologies, NY, USA). One microgram of mRNA was reverse transcribed to cDNA according to the manufacturer's instructions. The expression levels of gene transcripts were determined using quantitative real-time PCR. The qRT-PCR mix contained optimal concentrations of primers, CDNA and SYBR Green PCR Master Mix (Life Technologies, NY, USA). Relative gene expression was normalized to $\beta$-actin and represented as fold change (Meruvu et al., 2016b). Primers' sequences are shown in table 1. 


\section{Western blot analysis}

Cell were lysed with RIPA lysis buffer (Cat\#9803, Cell signaling) plus 0.5 mM PMSF and Protein inhibitor tablets (Cat\#A32963, Pierce ${ }^{\mathrm{TM}}$, Thermo Scientific). The protein content of the lysate was determined using Bio-Rad Protein Assay (Bio-Rad) on a spectrophotometer. The same amount of isolated proteins were separated by SDS-PAGE in LI-COR running buffer with reduced reagent, and transferred to PVDF membrane. After the membrane was blocked with LI-COR blocking buffer, it was immunoblotted with primary antibody, including SIRT1 (Cat\#2028), SIRT2 (Cat\#D4S6J), SIRT3 (Cat\#5490), SIRT5 (Cat\#8779), SIRT6 (Cat\#12486), SIRT7 (Cat\#5360) or GAPDH (Cat\#2118S) from Cell Signaling Technologies, or SIRT4 (ab124521) and Total OXPHOS Rodent WB Antibody Cocktail (ab110413) from Abcam, or $\beta$-actin (Cat\#PA5-59497) and MTCO1 (Cat\#459600) from ThermoFisher Scientific. Finally, Goat anti-rabbit or anti-mouse IRDye 680 or IRDye 800 secondary antibodies were used for the detection and quantitation of immunoblots. Membranes were imaged using a LI-COR Odyssey scanner, and blots were analyzed by Image Studio Lite 5.0 analytical software (LI-COR, Lincoln, NE) as previously described (Meruvu et al., 2016a; Zhang and Choudhury, 2017).

\section{Oxidative stress assay}

Reactive oxygen species (ROS) levels were measured using 2', 7'-dichlorofluorescein diacetate (DCFDA) as substrate according to the manufacturer's instructions (Meruvu et al., 2016b; Zhang et al., 2015). Briefly, HepG2 cells were seeded in a 96-well plate knocked down by DLEU2 SiRNA for $96 \mathrm{~h}$ as described earlier. Cells were then incubated with $25 \mu \mathrm{M}$ DCFDA for $45 \mathrm{~min}$ at $37^{\circ} \mathrm{C}$ in dark conditions, and fluorescence increment 
was measured for 30 min using a SpectraMax microplate reader (Molecular Devices, CA, USA) at $485 \mathrm{~nm}$ excitation and $535 \mathrm{~nm}$ emission.

\section{Statistical analysis}

All data are presented as the mean \pm standard error (S.E.M). Comparison between groups was performed by using One-way ANOVA (> two groups, followed by Tukey's test) or Student's t-test (two groups). A P-value less than 0.05 was considered a statistically significant difference. Statistical analysis was performed using Prism 6.0.

Table1. Gene primers used in this study.

\begin{tabular}{|c|c|c|}
\hline Gene & Forward primer $\left(5^{\prime}-3^{\prime}\right)$ & Reverse primer (3'-5') \\
\hline hSirt1(F2) & TAGCCTTGTCAGATAAGGAAGGA & ACAGCTTCACAGTCAACTTTGT \\
\hline hSirt2 (F1) & ATCCACCGGCCTCTATGACAA & CGCATGAAGTAGTGACAGATGG \\
\hline hSirt3 (F1) & GACATTCGGGCTGACGTGAT & ACCACATGCAGCAAGAACCTC \\
\hline hSirt4 (F1) & GCTTTGCGTTGACTTTCAGGT & CCAATGGAGGCTTTCGAGCA \\
\hline hSirt5 (F1) & GCCATAGCCGAGTGTGAGAC & CAACTCCACAAGAGGTACATCG \\
\hline hSirt6 (F1) & CCCGGATCAACGGCTCTATC & GCCTTCACCCTTTTGGGGG \\
\hline hSirt7 (F1) & CGTCCGGAACGCCAAATAC & GACGCTGCCGTGCTGATT \\
\hline $\mathrm{h} \beta$-actin $(\mathrm{F} 1)$ & ACCTTCTACAATGAGCTGCG & CCTGGATAGCAACGTACATGG \\
\hline hDLEU2 (F1) & GCGGGTACTTATCTCCGACC & GTTTCCCAGTCGCTGTTCGT \\
\hline
\end{tabular}

ACKNOWLEDGEMENT: The work was supported by Morris L Lichtenstein Jr. Medical Research Foundation. We would like to acknowledge Dr. Catherine Powell, for 
collecting data for body weight and Dr. Raktima Bhattacharya for running some qPCR plates. We also thank Faith Upton for helping the model drawing.

AUTHOR CONTRIBUTIONS-All three authors intellectually put their effort to hypothesize, carry out, and interpreting the data. M. C. designed the experiments in coordination with J.Z. J. Z. and M.K performed of the experiments, M. K. wrote the manuscript with M. C. and J. Z.

\section{DECLARATION OF INTERESTS}

The authors declare no competing interests

\section{REFERENCES}

Abdelmalek MF, S.A., Guy C, et al. (2010). Increased fructose consumption is associated with fibrosis severity in patients with nonalcoholic fatty liver disease. Hepatology 51, 1961-1971.

An, T., Zhang, T., Teng, F., Zuo, J.C., Pan, Y.Y., Liu, Y.F., Miao, J.N., Gu, Y.J., Yu, N., Zhao, D.D., et al. (2017). Long non-coding RNAs could act as vectors for paternal heredity of high fat diet-induced obesity. Oncotarget 8, 47876-47889.

Bause, A.S., and Haigis, M.C. (2013). SIRT3 regulation of mitochondrial oxidative stress. Exp Gerontol 48, 634-639.

Bober, E., Fang, J., Smolka, C., lanni, A., Vakhrusheva, O., Krüger, M., and Braun, T. (2012). Sirt7 promotes adipogenesis by binding to and inhibiting Sirt1. BMC Proceedings 6, P57.

Borengasser, S.J., Lau, F., Kang, P., Blackburn, M.L., Ronis, M.J., Badger, T.M., and Shankar, K. (2011). Maternal obesity during gestation impairs fatty acid oxidation and mitochondrial SIRT3 expression in rat offspring at weaning. PLoS One 6, e24068.

Carrico, C., Meyer, J.G., He, W., Gibson, B.W., and Verdin, E. (2018). The Mitochondrial Acylome

Emerges: Proteomics, Regulation by Sirtuins, and Metabolic and Disease Implications. Cell Metab 27, 497-512.

Chang, T.C., Yu, D., Lee, Y.S., Wentzel, E.A., Arking, D.E., West, K.M., Dang, C.V., Thomas-Tikhonenko, A., and Mendell, J.T. (2008). Widespread microRNA repression by Myc contributes to tumorigenesis. Nat Genet 40, 43-50.

Charlton, M., Krishnan, A., Viker, K., Sanderson, S., Cazanave, S., McConico, A., Masuoko, H., and Gores, G. (2011). Fast food diet mouse: novel small animal model of NASH with ballooning, progressive fibrosis, and high physiological fidelity to the human condition. Am J Physiol Gastrointest Liver Physiol 301, G825834.

Chen, C.Q., Chen, C.S., Chen, J.J., Zhou, L.P., Xu, H.L., Jin, W.W., Wu, J.B., and Gao, S.M. (2013). Histone deacetylases inhibitor trichostatin A increases the expression of Dleu2/miR-15a/16-1 via HDAC3 in nonsmall cell lung cancer. Mol Cell Biochem 383, 137-148. 
Cheng, M.Y., Cheng, Y.W., Yan, J., Hu, X.Q., Zhang, H., Wang, Z.R., Yin, Q., and Cheng, W. (2016). SIRT6 suppresses mitochondrial defects and cell death via the NF-kappaB pathway in myocardial hypoxia/reoxygenation induced injury. Am J Transl Res 8, 5005-5015.

Choudhary, C., Kumar, C., Gnad, F., Nielsen, M.L., Rehman, M., Walther, T.C., Olsen, J.V., and Mann, M. (2009). Lysine acetylation targets protein complexes and co-regulates major cellular functions. Science $325,834-840$.

Choudhury, M., and Friedman, J.E. (2011). Obesity: childhood obesity--methylate now, pay later? Nat Rev Endocrinol 7, 439-440.

Choudhury, M., Jonscher, K.R., and Friedman, J.E. (2011). Reduced mitochondrial function in obesityassociated fatty liver: SIRT3 takes on the fat. Aging (Albany NY) 3, 175-178.

Chukijrungroat, N., Khamphaya, T., Weerachayaphorn, J., Songserm, T., and Saengsirisuwan, V. (2017). Hepatic FGF21 mediates sex differences in high-fat high-fructose diet-induced fatty liver. Am J Physiol Endocrinol Metab 313, E203-E212.

Della Vedova, M.C., Munoz, M.D., Santillan, L.D., Plateo-Pignatari, M.G., Germano, M.J., Rinaldi Tosi, M.E., Garcia, S., Gomez, N.N., Fornes, M.W., Gomez Mejiba, S.E., et al. (2016). A Mouse Model of DietInduced Obesity Resembling Most Features of Human Metabolic Syndrome. Nutr Metab Insights 9, 93102.

Dupont, C., Armant, D.R., and Brenner, C.A. (2009). Epigenetics: definition, mechanisms and clinical perspective. Semin Reprod Med 27, 351-357.

Fang, J., Ianni, A., Smolka, C., Vakhrusheva, O., Nolte, H., Kruger, M., Wietelmann, A., Simonet, N.G., Adrian-Segarra, J.M., Vaquero, A., et al. (2017). Sirt7 promotes adipogenesis in the mouse by inhibiting autocatalytic activation of Sirt1. Proc Natl Acad Sci U S A 114, E8352-E8361.

Feldman, J.L., Dittenhafer-Reed, K.E., and Denu, J.M. (2012). Sirtuin catalysis and regulation. J Biol Chem 287, 42419-42427.

Godfrey, K.M. (1998). Maternal regulation of fetal development and health in adult life. Eur J Obstet Gynecol Reprod Biol 78, 141-150.

Gonzalez-Rodriguez, P., Cantu, J., O'Neil, D., Seferovic, M.D., Goodspeed, D.M., Suter, M.A., and Aagaard, K.M. (2016). Alterations in expression of imprinted genes from the H19/IGF2 loci in a multigenerational model of intrauterine growth restriction (IUGR). Am J Obstet Gynecol 214, 625 e621625 e611.

Goran MI, U.S., Ventura EE (2013). High fructose corn syrup and diabetes prevalence: a global perspective. . Globe Public Health 8, 55-64.

Gotto AM Jr, B.G., Dailey GE III, et al (2006). The metabolic syndrome: a call to action. Coron Artery Dis 17, 77-80.

Hirschey, M.D., Shimazu, T., Goetzman, E., Jing, E., Schwer, B., Lombard, D.B., Grueter, C.A., Harris, C., Biddinger, S., Ilkayeva, O.R., et al. (2010). SIRT3 regulates mitochondrial fatty-acid oxidation by reversible enzyme deacetylation. Nature 464, 121-125.

Hirschey, M.D., Shimazu, T., Jing, E., Grueter, C.A., Collins, A.M., Aouizerat, B., Stancakova, A., Goetzman, E., Lam, M.M., Schwer, B., et al. (2011). SIRT3 deficiency and mitochondrial protein hyperacetylation accelerate the development of the metabolic syndrome. Molecular cell 44, 177-190. Horsthemke, B. (2018). A critical view on transgenerational epigenetic inheritance in humans. Nat Commun 9, 2973.

Jeninga, E.H., Schoonjans, K., and Auwerx, J. (2010). Reversible acetylation of PGC-1: connecting energy sensors and effectors to guarantee metabolic flexibility. Oncogene 29, 4617-4624.

Jones, H.N., Woollett, L.A., Barbour, N., Prasad, P.D., Powell, T.L., and Jansson, T. (2009). High-fat diet before and during pregnancy causes marked up-regulation of placental nutrient transport and fetal overgrowth in C57/BL6 mice. FASEB J 23, 271-278. 
Kendrick, A.A., Choudhury, M., Rahman, S.M., McCurdy, C.E., Friederich, M., Van Hove, J.L., Watson, P.A., Birdsey, N., Bao, J., Gius, D., et al. (2011). Fatty liver is associated with reduced SIRT3 activity and mitochondrial protein hyperacetylation. Biochem J 433, 505-514.

Kim, H.S., Patel, K., Muldoon-Jacobs, K., Bisht, K.S., Aykin-Burns, N., Pennington, J.D., van der Meer, R., Nguyen, P., Savage, J., Owens, K.M., et al. (2010). SIRT3 Is a Mitochondria-Localized Tumor Suppressor Required for Maintenance of Mitochondrial Integrity and Metabolism during Stress. Cancer Cell 17, 4152.

King, J.C. (2006). Maternal obesity, metabolism, and pregnancy outcomes. Annu Rev Nutr 26, 271-291. King, S.E., and Skinner, M.K. (2020). Epigenetic Transgenerational Inheritance of Obesity Susceptibility. Trends Endocrinol Metab 31, 478-494.

Klein, U., Lia, M., Crespo, M., Siegel, R., Shen, Q., Mo, T., Ambesi-Impiombato, A., Califano, A., Migliazza, A., Bhagat, G., et al. (2010). The DLEU2/miR-15a/16-1 cluster controls B cell proliferation and its deletion leads to chronic lymphocytic leukemia. Cancer Cell 17, 28-40.

Kong, X., Wang, R., Xue, Y., Liu, X., Zhang, H., Chen, Y., Fang, F., and Chang, Y. (2010). Sirtuin 3, a new target of PGC-1alpha, plays an important role in the suppression of ROS and mitochondrial biogenesis. PLoS One 5, e11707.

Kwon, D.N., Chang, B.S., and Kim, J.H. (2014). MicroRNA dysregulation in liver and pancreas of CMPNeu5Ac hydroxylase null mice disrupts insulin/PI3K-AKT signaling. Biomed Res Int 2014, 236385.

Lacal, I., and Ventura, R. (2018). Epigenetic Inheritance: Concepts, Mechanisms and Perspectives. Front Mol Neurosci 11, 292.

Lerner, M., Harada, M., Loven, J., Castro, J., Davis, Z., Oscier, D., Henriksson, M., Sangfelt, O., Grander, D., and Corcoran, M.M. (2009). DLEU2, frequently deleted in malignancy, functions as a critical host gene of the cell cycle inhibitory microRNAs miR-15a and miR-16-1. Exp Cell Res 315, 2941-2952. Li, Y. (2018). Epigenetic Mechanisms Link Maternal Diets and Gut Microbiome to Obesity in the Offspring. Front Genet 9, 342.

Liu, B., Che, W., Zheng, C., Liu, W., Wen, J., Fu, H., Tang, K., Zhang, J., and Xu, Y. (2013). SIRT5: a safeguard against oxidative stress-induced apoptosis in cardiomyocytes. Cell Physiol Biochem 32, 10501059.

Liu, X.J., Duan, N.N., Liu, C., Niu, C., Liu, X.P., and Wu, J. (2018). Characterization of a murine nonalcoholic steatohepatitis model induced by high fat high calorie diet plus fructose and glucose in drinking water. Lab Invest 98, 1184-1199.

Lombard, D.B., Tishkoff, D.X., and Bao, J. (2011). Mitochondrial sirtuins in the regulation of mitochondrial activity and metabolic adaptation. Handb Exp Pharmacol 206, 163-188.

Love, S., Mudasir, M.A., Bhardwaj, S.C., Singh, G., and Tasduq, S.A. (2017). Long-term administration of tacrolimus and everolimus prevents high cholesterol-high fructose-induced steatosis in C57BL/6J mice by inhibiting de-novo lipogenesis. Oncotarget 8, 113403-113417.

Luo, Y., Burrington, C.M., Graff, E.C., Zhang, J., Judd, R.L., Suksaranjit, P., Kaewpoowat, Q., Davenport, S.K., O'Neill, A.M., and Greene, M.W. (2016). Metabolic phenotype and adipose and liver features in a high-fat Western diet-induced mouse model of obesity-linked NAFLD. Am J Physiol Endocrinol Metab 310, E418-439.

Lustig RH, M.K., Noworolski SM, et al. (2016). Isocaloric fructose restriction and metabolic improvement in children with obesity and metabolic syndrome. . Obesity (Silver Spring) 24, 453-460.

Lv, M., Zhong, Z., Huang, M., Tian, Q., Jiang, R., and Chen, J. (2017). IncRNA H19 regulates epithelialmesenchymal transition and metastasis of bladder cancer by miR-29b-3p as competing endogenous RNA. Biochim Biophys Acta 1864, 1887-1899.

Ma, L., Bajic, V.B., and Zhang, Z. (2013). On the classification of long non-coding RNAs. RNA Biol 10, 925933. 
Mastrocola, R., Ferrocino, I., Liberto, E., Chiazza, F., Cento, A.S., Collotta, D., Querio, G., Nigro, D., Bitonto, V., Cutrin, J.C., et al. (2018). Fructose liquid and solid formulations differently affect gut integrity, microbiota composition and related liver toxicity: a comparative in vivo study. J Nutr Biochem 55, 185-199.

Mazo, A., Hodgson, J.W., Petruk, S., Sedkov, Y., and Brock, H.W. (2007). Transcriptional interference: an unexpected layer of complexity in gene regulation. J Cell Sci 120, 2755-2761.

Meruvu, S., Zhang, J., Bedi, Y.S., and Choudhury, M. (2016a). Mono-(2-ethylhexyl) phthalate induces apoptosis through miR-16 in human first trimester placental cell line HTR-8/SVneo. Toxicol In Vitro 31, 35-42.

Meruvu, S., Zhang, J., and Choudhury, M. (2016b). Mono-(2-ethylhexyl) Phthalate Increases Oxidative Stress Responsive miRNAs in First Trimester Placental Cell Line HTR8/SVneo. Chem Res Toxicol 29, 430435.

Michishita, E., Park, J.Y., Burneskis, J.M., Barrett, J.C., and Horikawa, I. (2005). Evolutionarily conserved and nonconserved cellular localizations and functions of human SIRT proteins. Mol Biol Cell 16, 46234635.

Milazzo, G., Mercatelli, D., Di Muzio, G., Triboli, L., De Rosa, P., Perini, G., and Giorgi, F.M. (2020). Histone Deacetylases (HDACs): Evolution, Specificity, Role in Transcriptional Complexes, and Pharmacological Actionability. Genes (Basel) 11.

Nakagawa, T., Lomb, D.J., Haigis, M.C., and Guarente, L. (2009). SIRT5 Deacetylates carbamoyl phosphate synthetase 1 and regulates the urea cycle. Cell 137, 560-570.

Nasrin, N., Wu, X., Fortier, E., Feng, Y., Bare, O.C., Chen, S., Ren, X., Wu, Z., Streeper, R.S., and Bordone, L. (2010). SIRT4 regulates fatty acid oxidation and mitochondrial gene expression in liver and muscle cells. J Biol Chem 285, 31995-32002.

Ng, M., Fleming, T., Robinson, M., Thomson, B., Graetz, N., Margono, C., Mullany, E.C., Biryukov, S., Abbafati, C., Abera, S.F., et al. (2014). Global, regional, and national prevalence of overweight and obesity in children and adults during 1980-2013: a systematic analysis for the Global Burden of Disease Study 2013. Lancet 384, 766-781.

Ngo, D.T.M., Sverdlov, A.L., Karki, S., Macartney-Coxson, D., Stubbs, R.S., Farb, M.G., Carmine, B., Hess, D.T., Colucci, W.S., and Gokce, N. (2019). Oxidative modifications of mitochondrial complex II are associated with insulin resistance of visceral fat in obesity. Am J Physiol Endocrinol Metab 316, E168E177.

Nita, M., and Grzybowski, A. (2016). The Role of the Reactive Oxygen Species and Oxidative Stress in the Pathomechanism of the Age-Related Ocular Diseases and Other Pathologies of the Anterior and Posterior Eye Segments in Adults. Oxid Med Cell Longev 2016, 3164734.

Perkel, J.M. (2013). Visiting "noncodarnia". Biotechniques 54, 301, 303-304.

Potthast, A.B., Heuer, T., Warneke, S.J., and Das, A.M. (2017). Alterations of sirtuins in mitochondrial cytochrome c-oxidase deficiency. PLoS One 12, e0186517.

Powell, C.A., and Choudhury, M. (2019). Advancing metabolism research to overcome low litter survival in metabolically stressed mice. Am J Physiol Endocrinol Metab 317, E261-E268.

Qiu, X., Brown, K., Hirschey, M.D., Verdin, E., and Chen, D. (2010). Calorie restriction reduces oxidative stress by SIRT3-mediated SOD2 activation. Cell Metab 12, 662-667.

Rinn, J.L., Kertesz, M., Wang, J.K., Squazzo, S.L., Xu, X., Brugmann, S.A., Goodnough, L.H., Helms, J.A., Farnham, P.J., Segal, E., et al. (2007). Functional demarcation of active and silent chromatin domains in human HOX loci by noncoding RNAs. Cell 129, 1311-1323.

Roseboom, T.J., van der Meulen, J.H., Ravelli, A.C., Osmond, C., Barker, D.J., and Bleker, O.P. (2001). Effects of prenatal exposure to the Dutch famine on adult disease in later life: an overview. Twin Res 4, 293-298. 
Salminen, A., Kaarniranta, K., and Kauppinen, A. (2013). Crosstalk between Oxidative Stress and SIRT1: Impact on the Aging Process. Int J Mol Sci 14, 3834-3859.

Samuelsson, A.M., Matthews, P.A., Argenton, M., Christie, M.R., McConnell, J.M., Jansen, E.H., Piersma, A.H., Ozanne, S.E., Twinn, D.F., Remacle, C., et al. (2008). Diet-induced obesity in female mice leads to offspring hyperphagia, adiposity, hypertension, and insulin resistance: a novel murine model of developmental programming. Hypertension 51, 383-392.

Singh, C.K., Chhabra, G., Ndiaye, M.A., Garcia-Peterson, L.M., Mack, N.J., and Ahmad, N. (2018). The Role of Sirtuins in Antioxidant and Redox Signaling. Antioxid Redox Signal 28, 643-661.

Soro-Arnaiz, I., Li, Q.O.Y., Torres-Capelli, M., Melendez-Rodriguez, F., Veiga, S., Veys, K., Sebastian, D., Elorza, A., Tello, D., Hernansanz-Agustin, P., et al. (2016). Role of Mitochondrial Complex IV in AgeDependent Obesity. Cell Rep 16, 2991-3002.

Sundaresan, N.R., Gupta, M., Kim, G., Rajamohan, S.B., Isbatan, A., and Gupta, M.P. (2009). Sirt3 blocks the cardiac hypertrophic response by augmenting Foxo3a-dependent antioxidant defense mechanisms in mice. J Clin Invest 119, 2758-2771.

Suter, M.A., Chen, A., Burdine, M.S., Choudhury, M., Harris, R.A., Lane, R.H., Friedman, J.E., Grove, K.L., Tackett, A.J., and Aagaard, K.M. (2012). A maternal high-fat diet modulates fetal SIRT1 histone and protein deacetylase activity in nonhuman primates. FASEB J 26, 5106-5114.

Sverdlov, A.L., Elezaby, A., Behring, J.B., Bachschmid, M.M., Luptak, I., Tu, V.H., Siwik, D.A., Miller, E.J., Liesa, M., Shirihai, O.S., et al. (2015). High fat, high sucrose diet causes cardiac mitochondrial dysfunction due in part to oxidative post-translational modification of mitochondrial complex II. J Mol Cell Cardiol 78, 165-173.

Taylor, P.D., and Poston, L. (2007). Developmental programming of obesity in mammals. Exp Physiol 92, 287-298.

Tseng, A.H., Shieh, S.S., and Wang, D.L. (2013). SIRT3 deacetylates FOXO3 to protect mitochondria against oxidative damage. Free Radic Biol Med 63, 222-234.

van Otterdijk, S.D., and Michels, K.B. (2016). Transgenerational epigenetic inheritance in mammals: how good is the evidence? FASEB J 30, 2457-2465.

Verdin, E., Hirschey, M.D., Finley, L.W., and Haigis, M.C. (2010). Sirtuin regulation of mitochondria: energy production, apoptosis, and signaling. Trends Biochem Sci 35, 669-675.

Wadhwa, P.D., Buss, C., Entringer, S., and Swanson, J.M. (2009). Developmental origins of health and disease: brief history of the approach and current focus on epigenetic mechanisms. Semin Reprod Med 27, 358-368.

Xu, B., Gong, X., Zi, L., Li, G., Dong, S., Chen, X., and Li, Y. (2019). Silencing of DLEU2 suppresses pancreatic cancer cell proliferation and invasion by upregulating microRNA-455. Cancer Sci 110, 16761685.

Yajnik, C.S., and Deshmukh, U.S. (2008). Maternal nutrition, intrauterine programming and consequential risks in the offspring. Rev Endocr Metab Disord 9, 203-211.

Zhang, J., and Choudhury, M. (2017). The plasticizer BBP selectively inhibits epigenetic regulator sirtuin during differentiation of $\mathrm{C} 3 \mathrm{H} 10 \mathrm{~T} 1 / 2$ stem cell line. Toxicol In Vitro 39, 75-83.

Zhang, J., Meruvu, S., Bedi, Y.S., Chau, J., Arguelles, A., Rucker, R., and Choudhury, M. (2015).

Pyrroloquinoline quinone increases the expression and activity of Sirt1 and -3 genes in HepG2 cells. Nutr Res 35, 844-849.

Zwaans, B.M., and Lombard, D.B. (2014). Interplay between sirtuins, MYC and hypoxia-inducible factor in cancer-associated metabolic reprogramming. Dis Model Mech 7, 1023-1032. 
Fig 1
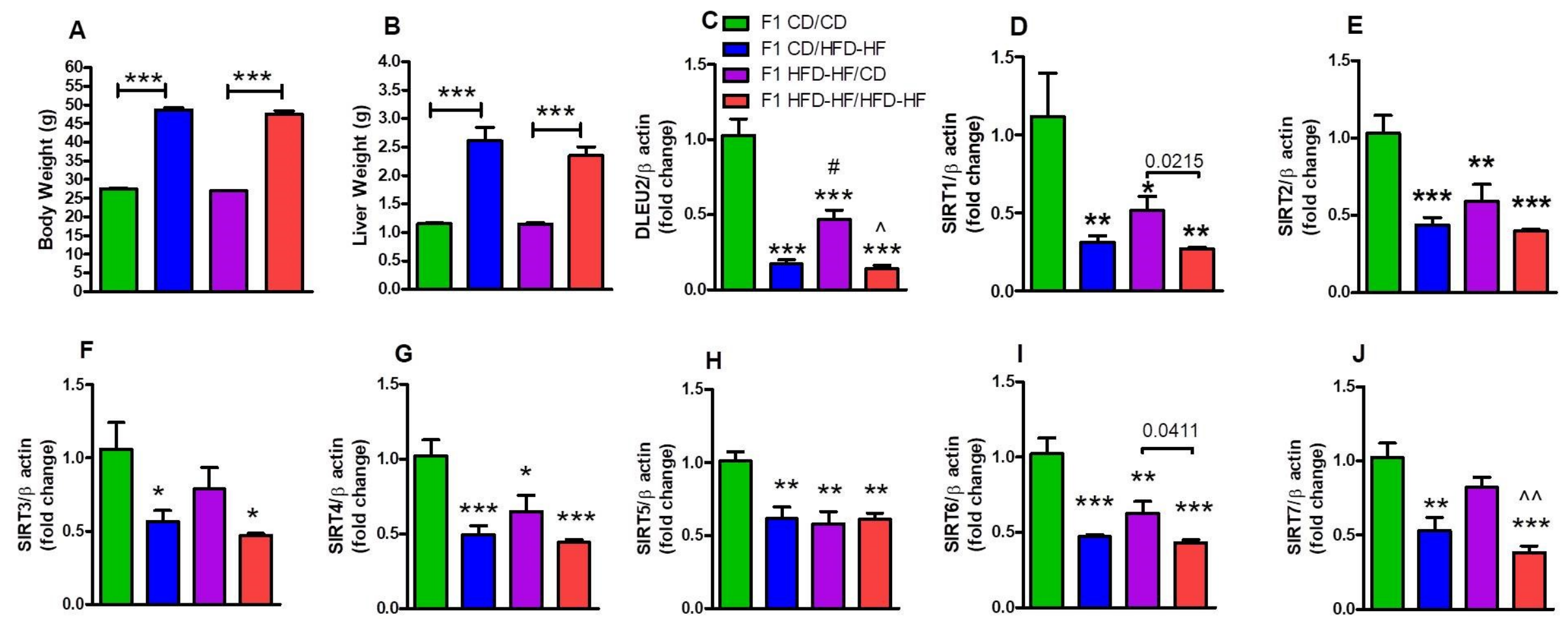
Fig 2
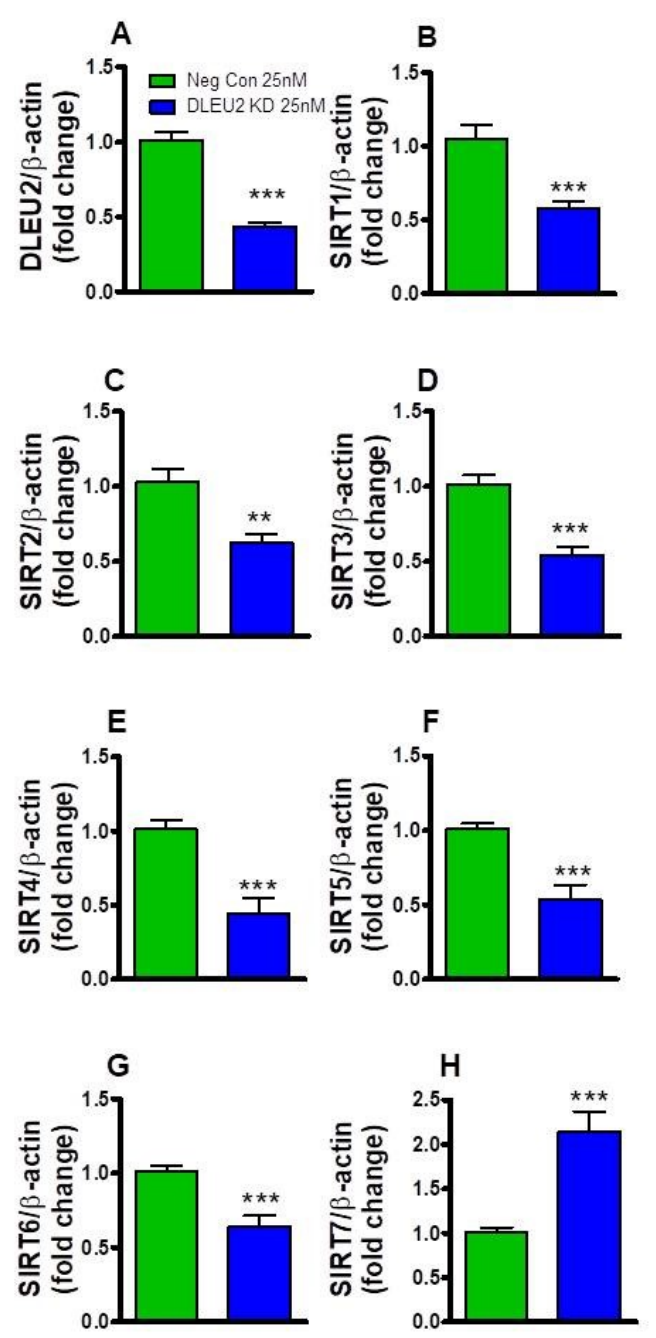

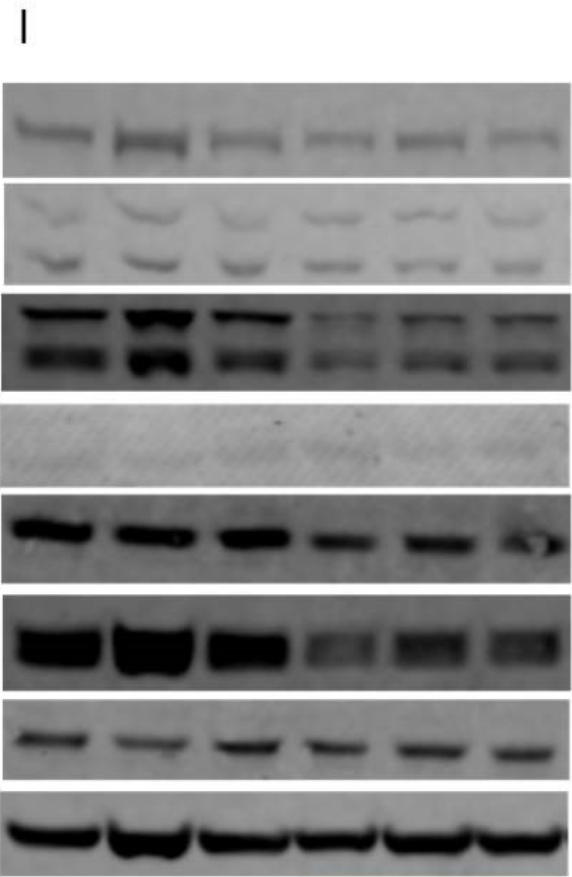

SIRT1-120KD

SIRT2-39, 43KD

SIRT3-28KD

SIRT4-32KD

SIRT5-30KD

SIRT6-42KD

SIRT7-45KD

$\beta$-actin-45KD

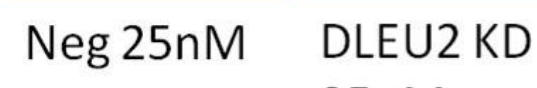

$25 \mathrm{nM}$
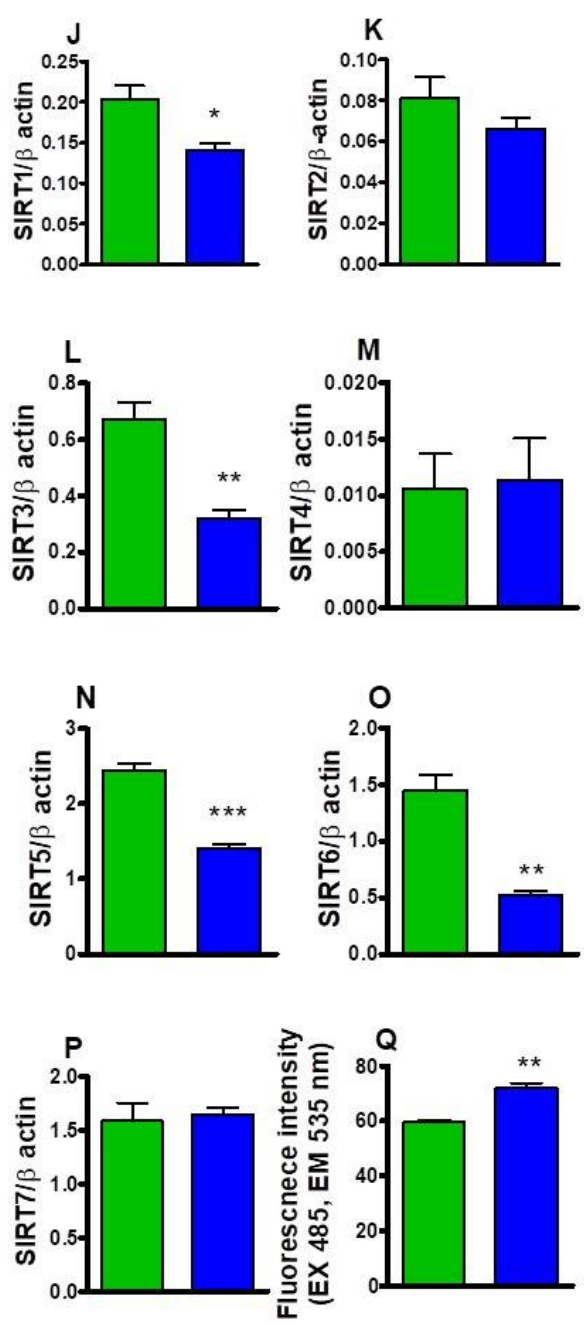
Fig 3

A

Neg 25nM DLEU2 KD 25nM
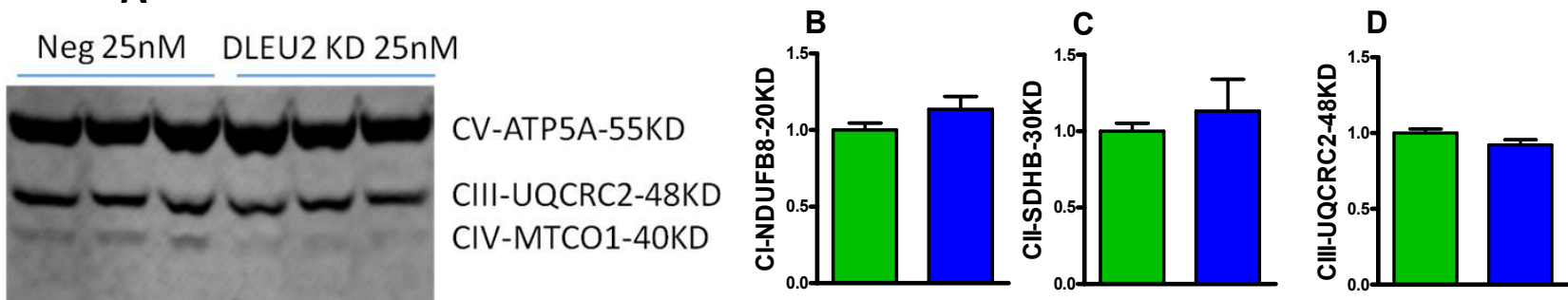

G

CII-SDHB-30KD

E F
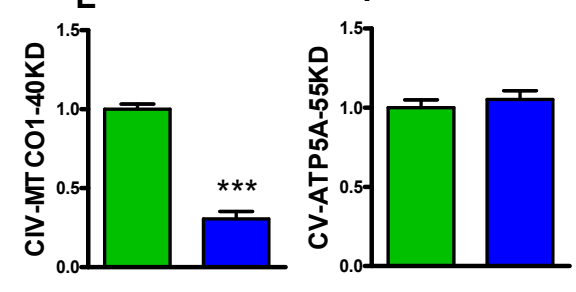

Neg Con 25nM

DDLEU2 KD $25 \mathrm{nM}$

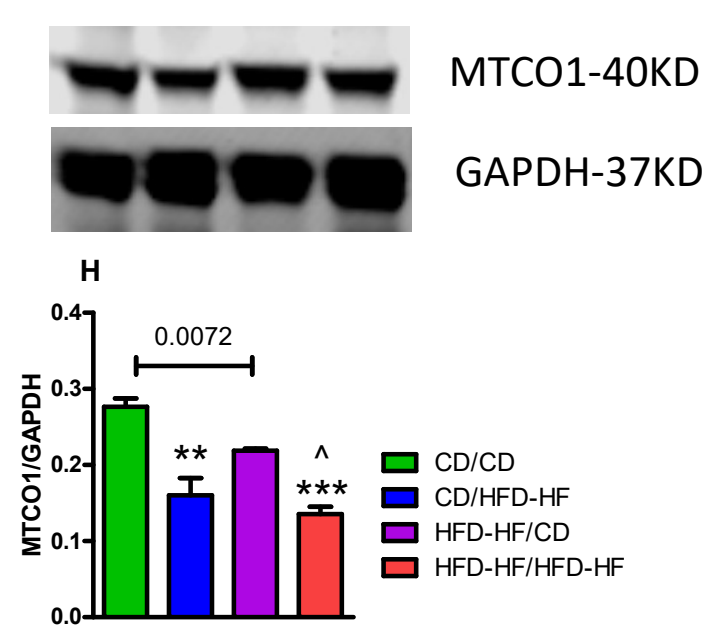


Fig 4

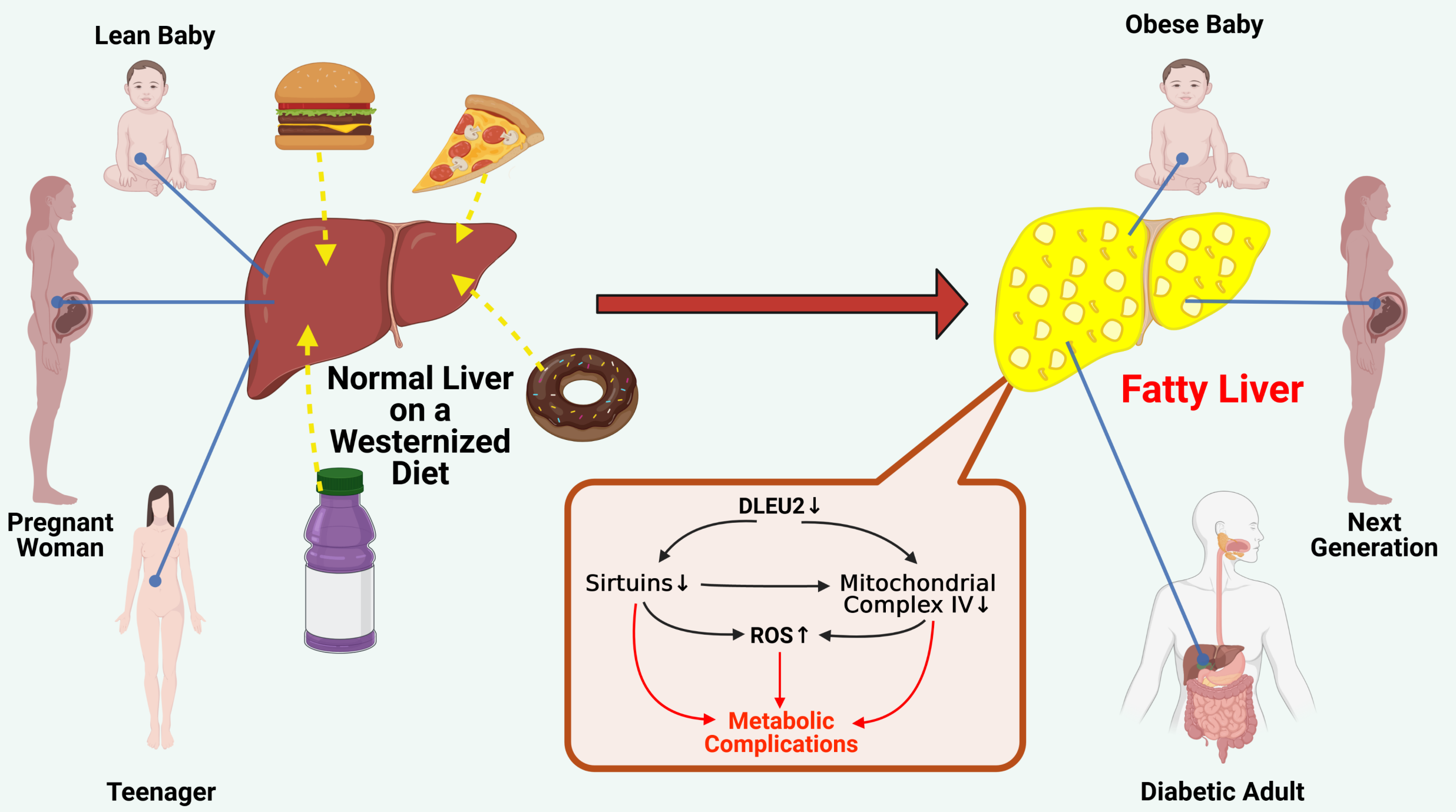

